OPEN ACCESS

Edited by: Leen Van Campenhout,

KU Leuven, Belgium

Reviewed by: Ganyu Gu, Agricultural Research Service (USDA), United States Dries Vandeweyer, KU Leuven, Belgium

*Correspondence: Annette Nygaard Jensen anyj@food.dtu.dk

Specialty section: This article was submitted to Food Microbiology, a section of the journal Frontiers in Microbiology

Received: 29 April 2020 Accepted: 19 June 2020 Published: 23 July 2020

Citation:

Jensen AN, Hansen SH and Baggesen DL (2020) Salmonella Typhimurium Level in Mealworms (Tenebrio molitor) After Exposure

to Contaminated Substrate.

Front. Microbiol. 11:1613. doi: 10.3389/fmicb.2020.01613

\section{Salmonella Typhimurium Level in Mealworms (Tenebrio molitor) After Exposure to Contaminated Substrate}

\author{
Annette Nygaard Jensen*, Sussie Hjort Hansen and Dorte Lau Baggesen \\ Division of Microbiology and Production, National Food Institute, Technical University of Denmark, Kongens Lyngby, \\ Denmark
}

Findings of viable Salmonella spp., which are important foodborne pathogens, are seemingly not reported in mealworms (Tenebrio molitor) for feed and food. Still, the bacterial load of mealworms is naturally high and includes members of the Enterobacteriaceae family to which Salmonella belong. This indicates that Salmonella may be able to thrive in mealworms if introduced into the production. Therefore, this study aimed to assess the quantitative level of Salmonella enterica serovar Typhimurium (ST) in mealworms over a 14-day course after exposure to substrate contaminated with ST levels from 1.7 to $7.4 \mathrm{log}$ CFU/g at start (i.e., day 0). The level of ST found in larvae was below the quantitative detection level ( 1 or 2 log CFU/g) on day 1 in larvae exposed to contamination levels of $1.7,3.4$, and $3.6 \mathrm{log} C F U / g$ opposed to contamination levels of 5.4, 5.6, and $7.4 \mathrm{log}$ CFU/g, respectively. The maximum level of ST detected in individual 1-g larvae samples was $5.8 \mathrm{log} \mathrm{CFU} / \mathrm{g}$, but the level varied among the triplicate samples from each sampling, and the highest average value was $5.3 \pm 0.3$. Beyond day 7 , only larvae exposed to the contamination level of $7.4 \mathrm{log}$ CFU/g were $>1.0 \mathrm{log}$ CFU/g in the triplicate samples. However, qualitative testing $(10 \mathrm{~g})$ showed the presence of ST in larvae until the end of the experiment on day 14 except for the lowest contamination level of $1.7 \mathrm{log}$ CFU/g. Parallel testing of surface disinfected larvae indicated that some larvae may be ST-positive due to Salmonella residing on the surface only. Still, any detection of Salmonella is of concern from a food safety perspective. In substrate with contamination levels below $3.6 \log \mathrm{CFU} / \mathrm{g}$, the level of ST was below the quantitative detection limit within a few days. Still, ST was detected until the end of experiment on day 14 except for the lowest contamination level of $1.7 \mathrm{log} \mathrm{CFU} / \mathrm{g}$. This study indicates the importance of avoiding introduction of Salmonella into the production, e.g., via contaminated substrate in order to avoid Salmonella-positive larvae as they remained positive for at least 14 days (except at the lowest contamination level).

Keywords: Tenebrio molitor, Salmonella, contamination level, persistence, food safety 


\section{INTRODUCTION}

The interest in rearing of edible insects as a new valuable source of food and feed rich in proteins has increased significantly in the Western world following the publication 'Edible insects Future prospects for food and feed security' from the Food and Agriculture Organization (FAO) in 2013 (van Huis et al., 2013). As for any other food and feed production chain, it is important to assess and control potential hazards associated to the production of insects and products derived thereof (Schlüter et al., 2016; van der Fels-Klerx et al., 2018; Raheem et al., 2019; Cappelli et al., 2020; Imathiu, 2020). Accordingly, The European Food Safety Authority (EFSA) prepared a risk profile related to production and consumption of insects as food and feed (EFSA Scientific Committee, 2015). Despite a scarcity of knowledge at that time about the specific risks associated to insects as a new form of mini-livestock, this risk profile emphasized the importance of hygienic conditions of the substrate and need for assessing the specific risks especially if other than food and feed-grade substrates are used (which is banned at present by EU regulation).

The EFSA risk profile also provided a list of insect species with high farming potential, which included the yellow mealworm (Tenebrio molitor) (EFSA Scientific Committee, 2015). As no appreciable consumption of mealworms took place in EU before May 15, 1997, mealworms are considered as Novel Foods by EU Regulation (EU) No 2015/2283 implying the requirement of authorization from EFSA before commercialization. Further, the general EU food law [Regulation (EC) No 178/2002] specifies that food and feed shall not be placed on market if unsafe. This also applies for insects even though the European Regulation (EC) No 2073/2005 on microbiological criteria for foodstuffs has set no specific criteria for mealworms (yet).

Due to a naturally high bacterial load of around $8 \log \mathrm{CFU} / \mathrm{g}$ in T. molitor including opportunistic human pathogens, heat treatment or other processing is recommended to reduce the bacterial load before consumption (EFSA Scientific Committee, 2015; Schlüter et al., 2016; Stoops et al., 2016; Vandeweyer et al., 2017; Osimani et al., 2018; Wynants et al., 2018; Garofalo et al., 2019). The Enterobacteriaceae family is one of the highly represented bacterial groups in T. molitor larvae, and although Salmonella spp. belongs to this family, no findings of viable Salmonella has to our knowledge been reported yet (Grabowski and Klein, 2017; Vandeweyer et al., 2017; Osimani et al., 2018). This may be due to the use of feed-grade substrates as several insects as well as mealworms have vector potential for carrying Salmonella after exposure (Skov et al., 2004; Roche et al., 2009; Blazar et al., 2011; Nordentoft et al., 2017; Crippen et al., 2012, 2018; Wynants et al., 2019). Another explanation may be a potential capability of insects to fight incoming pathogens ( $\mathrm{Wu}$ et al., 2018; Jo et al., 2019; Keshavarz et al., 2020).

As Salmonella spp. are important foodborne pathogens with $>91,000$ reported salmonellosis cases in EU annually, significant efforts are generally made to prevent their presence in feed and food production chains, e.g., stated by Regulation (EC) No 2160/2003 (European Food Safety Authority [EFSA], and European Centre for Disease Prevention, and Control [ECDC],
2019). A broad range of animals can carry Salmonella spp. and often without any symptoms, causing fecal excretion of Salmonella into the environment and potential transmission to other animals, crops and water reservoirs. Consequently, contaminated substrate, insufficient hygienic measures or lack of biosecurity for preventing entrance of infected wild insects, rodents and pets may introduce Salmonella spp. into insect production facilities.

For documenting the absence of Salmonella spp. in food, qualitative testing of the presence of Salmonella spp. by preenrichment of 10-g or 25-g samples is usually applied (e.g., ISO, 2017 6579-1). However, the level of Salmonella contamination is also of interest as dose-response modeling of Salmonella using outbreak data indicated that both the risk of infection and the risk of illness given infection increase with dose (Teunis et al., 2010). The dose response model found that the number of CFUs causing infection or illness in 50\% (ID50) of exposed people were 7 and $36 \mathrm{CFU}$, respectively. Noteworthy, the bacterial cell count obtained from a sample, will depend on the ability to retrieve cells adequately from the sample matrix, which is affected by the nature of the matrix (e.g., fat content, physical structure) and the homogenization method applied (Rohde et al., 2015). Accordingly, proper sample treatment is important for enumerating the actual number of cells present and avoiding underestimation or false negative results. Still, there seem to be no suggestions for or evaluations of a proper and standardized treatment method for mealworm samples before enumeration or detection of the bacterial content.

One recent study assessed how the presence of Salmonella sp. during rearing of mealworms affected the survival in substrate and transmission to larvae (Wynants et al., 2019). The time course was here 7 days and it is of interest to see if longer time, e.g., will support clearance of Salmonella, which is relevant concerning mitigation options. In addition, more contamination levels, higher frequency of samplings and quantitative testing may help to a better understanding of the fate of Salmonella after introduction into a mealworm production site as well as the importance of the Salmonella contamination level.

In this study, we firstly aimed to test the ability to recover Salmonella Typhimurium from mealworm samples with the applied detection method. Secondly, we aimed to assess the quantitative level of $S$. Typhimurium in disinfected and nondisinfected mealworms over a 14-day course after exposure to substrate contaminated with different levels of Salmonella at start, as well as the fate of Salmonella in the substrate.

\section{MATERIALS AND METHODS}

\section{Mealworms}

Mealworms (Tenebrio molitor, L.) provided by the Danish Technological Institute (Aarhus, DK) were reared in-house at the National Food Institute, Technological University of Denmark (DTU) (Kgs. Lyngby, DK) on a flour-based dry substrate provided by Adival A/S (Billund, DK) and spent grain acquired from DTU Brewery (Kgs. Lyngby, DK) as wet feed. The rearing 
room had a temperature of $26.5 \pm 0.6^{\circ} \mathrm{C}$ and a relative humidity of $55.0 \pm 3.6 \%$.

\section{Salmonella Typhimurium Contamination Strain}

A Danish strain of Salmonella enterica serovar Typhimurium DT12 (S. Typhimurium or ST) previously made resistant to rifampicin (Jensen et al., 2006) was used to contaminate the substrate in an experimental laboratory study. A colony from an overnight (o.n.) plate-spread on tryptic soy agar with sheep blood (TSASB) (98763, SSI Diagnostica, Copenhagen, Denmark) was transferred into $10 \mathrm{~mL}$ Buffered Peptone Water (CM1049, Oxoid, DK) and incubated o.n. at $37^{\circ} \mathrm{C}$. A 10 -fold dilution series of the o.n. culture was prepared in $0.9 \% \mathrm{NaCl}$ solution to reach expected contamination levels ranging from approximately 2 to $7 \log \mathrm{CFU} / \mathrm{g}$, and $0.1 \mathrm{~mL}$ of each dilution was plate-spread onto TSASB to determine the exact concentration of ST.

\section{Quantitative Estimation of S. Typhimurium}

To estimate the level of $S$. Typhimurium in larvae, $1 \mathrm{~g}$ of larvae was sampled and added $1 \mathrm{~mL} 0.9 \% \mathrm{NaCl}$ solution before homogenization in-tube ( $12 \mathrm{~mL}$ round-bottomed tube) by grinding with a sterile Thomas pestle tissue grinder made of teflon and with at stainless steel shaft (Thomas Scientific, Swedesboro, NJ, United States). Additional $8 \mathrm{~mL}$ saline was added to the tube (i.e., $10^{-1}$ dilution) before vortexing and preparation of a 10 -fold dilution series in $0.9 \% \mathrm{NaCl}$. Appropriate dilutions were spread onto Nutrient Agar (NA) plates with $50 \mu \mathrm{g} / \mathrm{mL}$ rifampicin (R3501, Sigma-Aldrich Chemie, DE) $\left(\mathrm{NA}^{\text {rif }}\right)$. For samples expected to have concentrations $<100 \mathrm{CFU} / \mathrm{g}, 1 \mathrm{~mL}$ of the $10^{-1}$ dilution was distributed onto three agar plates to reach a detection limit of $10 \mathrm{CFU} / \mathrm{g}$, otherwise $0.1 \mathrm{~mL}$ was plated.

Substrate samples of $1 \mathrm{~g}$ were handled similarly, except that the homogenization step was performed simply by vortexing $15 \mathrm{~s}$.

The agar plates were incubated o.n. at $37^{\circ} \mathrm{C}$ before enumeration of ST presumptive colonies. Suspect colonies were verified by sub-culturing onto indicative plates as well as NA and $\mathrm{NA}^{\text {rif }}$ plates and agglutination serotyping as described in Section "Qualitative Detection of $S$. Typhimurium."

\section{Qualitative Detection of S. Typhimurium}

Qualitative detection of $S$. Typhimurium in homogenized larvae and substrate samples $(10 \mathrm{~g})$ was done by addition of $90 \mathrm{~mL}$ of buffered peptone water (BPW) (94515, SSI Diagnostica) before o.n. pre-enrichment at $37^{\circ} \mathrm{C}$. The $10 \mathrm{~g}$ larvae was homogenized by grinding with a pestle tissue grinder as above, but in a $50 \mathrm{~mL}$ tube added $2 \mathrm{~mL} \mathrm{BPW}$, and with transfer of the larvae material to a $150 \mathrm{~mL}$ cup by flushing the tube with additional $88 \mathrm{~mL}$ BPW in total. The o.n. culture was spotted onto modified semi-solid Rappaport-Vassiliadis (MSRV) agar (CM0910 and SR0161, Oxoid, DK) in three droplets (approx. $0.1 \mathrm{~mL}$ ). After o.n. incubation at $41.5^{\circ} \mathrm{C}$, material from presumptive Salmonella swarming zones was sub-cultured onto indicative plates Brilliant Green Agar plates (PO5033A, Oxoid) and Xylose Lysine Deoxycholate (XLD) agar plates (PO5057A, Oxoid) and incubated at $37^{\circ} \mathrm{C}$ o.n.

The ST contamination strain was verified by slide agglutination of colony material from the indicative plates with O4 (23839, SSI Diagnostica, DK) and O5 (40272, SSI Diagnostica) antiserum according to the Kauffmann-White scheme (Grimont and Weill, 2007) as well as confirmation of the resistance marker by sub-culturing onto NA and $\mathrm{NA}^{\text {rif. }}$.

\section{Detection Limit for S. Typhimurium in Mealworms}

The ability to detect Salmonella in mealworms was assessed by testing larvae artificially contaminated with specified levels of $S$. Typhimurium. A ST suspension (in duplicate) was prepared as described in Section "Salmonella Typhimurium Contamination Strain" and diluted tenfold. Then $100 \mu \mathrm{l}$ of each dilution was added to 1-g larvae samples to reach Salmonella concentrations from 1 to $10^{5} \mathrm{CFU} / \mathrm{g}$ larvae. The added cells were allowed to settle on the larvae for $10 \mathrm{~min}$, while kept at $5^{\circ} \mathrm{C}$ to avoid growth of the added cells and to calm the larvae before quantification of Salmonella as described in Section "Quantitative Estimation of $S$. Typhimurium."

Additional, qualitative detection of Salmonella was performed as described in Section "Qualitative Detection of S. Typhimurium." Larvae samples of $10 \mathrm{~g}$ were added ST in numbers from approximate $<1$ to $100 \mathrm{CFU}$ from appropriate dilutions of the suspension ( $100 \mu \mathrm{l}$ was added).

\section{Experimental Exposure of Mealworms to Salmonella-Contaminated Substrate}

In two successive experiments, 50-60 days old larvae close to the stage of pupation were exposed to substrate experimentally contaminated with different concentrations of $S$. Typhimurium at start of the experiment. Each experimental period lasted for 14 days. The experimental trays with larvae were placed in a separate incubator in the laboratory to avoid accidental contamination of the rearing room at temperatures of $25.5 \pm 0.3^{\circ} \mathrm{C}$ and $25.6 \pm 0.1^{\circ} \mathrm{C}$ and a $\mathrm{RH}$ of $55.9 \pm 6.1 \%$ and $39.6 \pm 4.6 \%$ in trials 1 and 2 , respectively.

\section{Experimental Trial 1}

For the experimental set-up, larvae with an average weight of $126 \pm 20 \mathrm{mg}$ were sieved to remove the substrate provided during rearing. Two days prior to exposure on day zero, larvae, and fresh flour-based dry substrate (Adival A/S) were allocated in a $1: 1$ ratio of $150 \mathrm{~g}$ into each of 4 aluminum foil trays $(19 \mathrm{~cm} \times 12.5 \mathrm{~cm} \times 4.5 \mathrm{~cm}, 1.1 \mathrm{~L})$. One additional tray was added substrate only (150 g).

On day zero, $1.7 \mathrm{~mL}$ of appropriate dilutions of the $S$. Typhimurium suspension was added to $50 \mathrm{~mL}$ Greiner tubes each containing $20 \mathrm{~g}$ spent grain (DTU Brewery). After thorough vortexing, the contaminated spent grain was transferred to the aluminum foil trays with larvae for estimated final ST concentrations of approximately 2, 4, and $6 \log$ CFU per $g$ of substrate in each tray, respectively, see Table 1. Additionally, contaminated spent grain was added to the tray with substrate 
TABLE 1 | Set-up for experimental exposure of mealworm to Salmonellacontaminated substrate.

\begin{tabular}{lllll}
\hline & \multicolumn{2}{c}{$\begin{array}{c}\text { Salmonella contamination level } \\
\text { (expected log CFU/g) }\end{array}$} & \\
\cline { 2 - 4 } Tray & Description & 1st trial & 2nd trial & Tray content ${ }^{\text {b }}$ \\
\hline 1 & Low & 2 & 3 & Larvae: substrate (1:1) \\
2 & Medium & 4 & 5 & Larvae: substrate $(1: 1)$ \\
3 & High & 6 & 7 & Larvae: substrate $(1: 1)$ \\
4 & Neg. control & 0 & 0 & Larvae: substrate $(1: 1)$ \\
5 & Control & 4 & 5 & Substrate
\end{tabular}

appropriate dilutions of the S. Typhimurium suspension were added via $20 \mathrm{~g}$ spent grain to reach an 'expected' final contamination level in the substrate at start of the experiment. ${ }^{b}$ Larvae were mixed with fresh dry substrate in a 1:1 ratio of $150 \mathrm{~g}$ 2 days prior to contamination with Salmonella.

only (4 log CFU/g of substrate) referred to as control, while $20 \mathrm{~g}$ uncontaminated spent grain was added to a tray with larvae as a negative control for cross-contamination between trays. During the experimental period, the larvae were provided water by addition of $20 \mathrm{~g}$ spent grain after each sampling.

\section{Experimental Trial 2}

The set-up was similar to the first trial but with the following modifications. The average larvae weight was $131 \pm 9 \mathrm{mg}$. The fixed volume the of $S$. Typhimurium suspension used to contaminate the spent grain was reduced to $1 \mathrm{~mL}$ and the contamination level was based on the results of the first trial increased to estimated final ST concentrations of approximately 3, 5, and $7 \log \mathrm{CFU}$ per $\mathrm{g}$ of substrate, respectively, see Table 1. The control tray without larvae was added 5 log CFU per $g$ of substrate.

\section{Sampling of Larvae and Substrate}

The first sampling was performed approximately 10 min after addition of spent grain, referred to as day 0 . During each experimental period, samplings were performed on day 0,1 , $2,5,7,9,12$, and 14 or until a sample rendered test-negative by qualitative testing. The negative controls were sampled days 0,7 , and 14 .

From each tray, $1 \mathrm{~g}$ of larvae was transferred to a round-bottomed $12 \mathrm{~mL}$ tube with a sterile tweezer $(n=6)$ for quantitative detection of $S$. Typhimurium (see section "Quantitative Estimation of S. Typhimurium"). Half of the larvae samples from each sampling was surface disinfected before testing (see section "Surface Disinfection of Larvae"). Following a negative test result by the quantitative testing approach, $10 \mathrm{~g}$ of larvae was transferred to a $50 \mathrm{~mL}$ Greiner tube for qualitative testing for the presence of ST (see section "Qualitative Detection of $S$. Typhimurium").

Substrate samples of $1 \mathrm{~g}$ mainly consisting of the flour-based substrate (leaving out spent grain residues) were collected from each tray with a plastic spoon and transferred to a $50 \mathrm{~mL}$ Greiner tube $(n=3)$ for quantitative detection of ST (see section "Quantitative Estimation of S. Typhimurium"). As for larvae, a 10 -g sample was collected for qualitative testing when the preceding sample was test negative.

\section{Surface Disinfection of Larvae}

To remove Salmonella present on the surface of larvae, $1 \mathrm{~g}$ larvae samples were collected into a tube and added $3 \mathrm{~mL}$ of $70 \% \mathrm{w} / \mathrm{w}$ ethanol and vortexed for $10 \mathrm{~s}$ before removal of the ethanol. The larvae were left for 2 min before being rinsed twice with $5 \mathrm{~mL}$ Milli Q water.

This method had been tested prior to this study (data not shown). Shortly, individual larva was submerged in a suspension of the ST strain (approx. $\left.10^{7} \mathrm{CFU} / \mathrm{mL}\right)$, then $1 \mathrm{~g}$ larvae $(n=3)$ were pooled and disinfected by the method described above before individual larva was pour plated in $25 \mathrm{~mL} \mathrm{NA}^{\text {rif }}$ and incubated overnight at $37^{\circ} \mathrm{C}$.

\section{Statistical Analyses}

The quantitative detection of ST in larvae was compared with the concentration of ST added (Table 2) by pairwise $t$-test in GraphPad Prism version 8.3.1 for Windows, GraphPad Software, San Diego, CA, United States, www.graphpad.com (RRID:SCR_002798). For substrate, ST counts for each contamination level were compared between sampling moments (Table 3) using repeated measures ANOVA in GraphPad Prism. When counts were below the detection limit applied, i.e., 1 or $2 \log \mathrm{CFU} / \mathrm{g}$ depending on the expected count, a value representing half of the detection limit, i.e., 0.5 or $1.0 \log$ $\mathrm{CFU} / \mathrm{g}$, respectively, was chosen as a value to be included in the statistical analysis. Qualitative testing was applied following a sampling with a ST count below the detection limit, where values of 0.0 and -0.3 were assigned for positive and negative results, respectively.

\section{RESULTS}

\section{Detection Limit for S. Typhimurium in Mealworms}

Colonies of the $S$. Typhimurium contamination strain on $\mathrm{NA}^{\text {rif }}$ agar plates were counted based on morphology of colonies verified to be the contamination strain as described in Section "Quantitative Estimation of S. Typhimurium" and Section

TABLE 2 | Quantitative detection level for S. Typhimurium in mealworms.

\begin{tabular}{|c|c|c|c|}
\hline \multicolumn{4}{|c|}{ ST in mealworm samples (log CFU/g) } \\
\hline \multicolumn{2}{|c|}{ 1st trial } & \multicolumn{2}{|c|}{ 2nd trial } \\
\hline Cells added ${ }^{a}$ & Cells detected & Cells added ${ }^{\mathrm{a}}$ & Cells detected \\
\hline 5.01 & 4.90 & 5.21 & 5.10 \\
\hline 4.01 & 4.00 & 4.21 & 4.08 \\
\hline 3.01 & 2.62 & 3.21 & 2.60 \\
\hline 2.01 & $2.15^{b}$ & 2.21 & $2.20^{\mathrm{b}}$ \\
\hline 1.01 & $N D^{C}$ & 1.21 & $1.70^{\mathrm{C}}$ \\
\hline 0.01 & $N^{C}$ & 0.21 & $N^{c}$ \\
\hline
\end{tabular}

${ }^{a}$ Addition of $100 \mu \mathrm{L}$ ST cell suspension (10-fold dilution series) to $1 \mathrm{~g}$ mealworm. ${ }^{b}$ Weighted average of CFU/g for plating of $100 \mu \mathrm{L}$ (in duplicate) and $1 \mathrm{~mL}$ of the $10^{-1}$ dilution. ${ }^{c}$ Plating of $1 \mathrm{~mL}$ of the $10^{-1}$ dilution ND, none detected. 
TABLE 3 | Level of Salmonella Typhimurium found in substrate, larvae and disinfected larvae over a 14 days period after a single contamination event of substrate with ST at start.

\begin{tabular}{|c|c|c|c|c|c|c|c|c|c|}
\hline \multirow[t]{3}{*}{ Sample } & \multirow{3}{*}{$\begin{array}{c}\text { Salmonella } \\
\text { added }^{\mathrm{a}} \\
(\log \mathrm{CFU} / \mathrm{g})\end{array}$} & \multicolumn{8}{|c|}{ S. Typhimurium log CFU/g ${ }^{b}$} \\
\hline & & \multicolumn{8}{|c|}{ Day after contamination of substrate } \\
\hline & & 0 & 1 & 2 & 5 & 7 & 9 & 12 & 14 \\
\hline \multirow[t]{6}{*}{ Larvae disinfected } & 1.7 & $0.5 \pm 0.9$ & $<1.0$ & $<1.0$ & - & - & - & - & - \\
\hline & 3.4 & $1.4 \pm 1.2$ & $1.1 \pm 1.9$ & Neg & Pos & - & Pos & Neg & - \\
\hline & 3.6 & $2.2 \pm 0.4$ & $<2.0$ & $<2.0$ & - & - & Neg & - & Neg \\
\hline & 5.4 & $3.8 \pm 0.5$ & $<2.0$ & $1.6 \pm 2.7$ & $1.0 \pm 1.7$ & $<1.0$ & $<1.0$ & Pos & Neg \\
\hline & 5.6 & $4.1 \pm 0.3$ & $<2.0$ & $<2.0$ & $0.7 \pm 1.2$ & $<2.0$ & Pos & $<2.0$ & Neg \\
\hline & 7.4 & $5.3 \pm 0.3$ & $2.0 \pm 1.8$ & $2.2 \pm 2.0$ & $<1.0$ & $2.3 \pm 2.4$ & $<2.0$ & $<1.0$ & Pos \\
\hline \multirow[t]{7}{*}{ Larvae } & Control & Neg & - & - & - & Neg & - & - & Neg \\
\hline & 1.7 & $0.8 \pm 1.4$ & $<1.0$ & $\mathrm{Neg}$ & $\mathrm{Neg}$ & Neg & - & - & - \\
\hline & 3.4 & $1.5 \pm 0.5$ & $<1.0$ & Neg & Pos & - & Pos & Pos & Pos \\
\hline & 3.6 & $3.0 \pm 0.4$ & $<2.0$ & Pos & Pos & Pos & Pos & Pos & Pos \\
\hline & 5.4 & $3.8 \pm 1.3$ & $2.4 \pm 2.1$ & $<2.0$ & $2.1 \pm 0.5$ & $1.2 \pm 2.1$ & $<1.0$ & Pos & Pos \\
\hline & 5.6 & $5.0 \pm 0.1$ & $2.4 \pm 2.2$ & $2.0 \pm 3.5$ & Pos & Pos & Pos & Pos & Pos \\
\hline & 7.4 & $5.2 \pm 0.5$ & $2.1 \pm 1.8$ & $<2.0$ & $3.1 \pm 0.5$ & $3.1 \pm 1.2$ & $2.3 \pm 2.2$ & $2.3 \pm 0.3$ & $1.9 \pm 0.3$ \\
\hline \multirow[t]{7}{*}{ Substrate with larvae } & Control & Neg & - & - & - & Neg & - & - & Neg \\
\hline & 1.7 & $0.9 \pm 1.5$ & $0.7 \pm 1.2$ & $<1.0$ & - & Neg & - & - & - \\
\hline & 3.4 & $1.6 \pm 1.4$ & $<1.0$ & Neg & Pos & - & Pos & Pos & Pos \\
\hline & 3.6 & $2.5 \pm 2.2$ & $0.7 \pm 1.2$ & $<2.0$ & - & Pos & Pos & - & Pos \\
\hline & 5.4 & $4.1 \pm 0.7^{\mathrm{AC}}$ & $1.4 \pm 1.2^{\mathrm{BC}}$ & $<2.0^{\mathrm{AB}}$ & $3.7 \pm 0.4^{\mathrm{AC}}$ & $3.2 \pm 0.3^{C D}$ & $1.8 \pm 0.5^{\mathrm{BD}}$ & $2.5 \pm 0.4^{\mathrm{BCD}}$ & $2.3 \pm 0.2^{\mathrm{AB}}$ \\
\hline & 5.6 & $4.7 \pm 0.8^{\mathrm{AB}}$ & $3.3 \pm 0.5^{\mathrm{A}}$ & $2.5 \pm 0.4^{\mathrm{B}}$ & $2.1 \pm 1.8^{\mathrm{AB}}$ & $3.0 \pm 1.2^{\mathrm{AB}}$ & $2.8 \pm 0.8^{\mathrm{B}}$ & $2.0 \pm 2.0^{\mathrm{AB}}$ & $2.0 \pm 2.0^{\mathrm{AB}}$ \\
\hline & 7.4 & $6.0 \pm 0.4^{\mathrm{AC}}$ & $3.4 \pm 0.2^{\mathrm{AB}}$ & $<2.0^{\mathrm{C}}$ & $3.8 \pm 0.1^{\mathrm{AB}}$ & $4.6 \pm 0.5^{\mathrm{AB}}$ & $3.7 \pm 0.1^{\mathrm{B}}$ & $3.5 \pm 0.2^{\mathrm{B}}$ & $3.7 \pm 0.1^{\mathrm{B}}$ \\
\hline \multirow[t]{2}{*}{ Substrate without larvae } & 3.4 & $1.7 \pm 1.5$ & $<2.0$ & $<2.0$ & - & Pos & Pos & Pos & Pos \\
\hline & 5.4 & $4.5 \pm 0.5$ & $<2.0$ & $<2.0$ & $0.4 \pm 0.8$ & $0.3 \pm 0.6$ & Pos & Pos & Pos \\
\hline
\end{tabular}

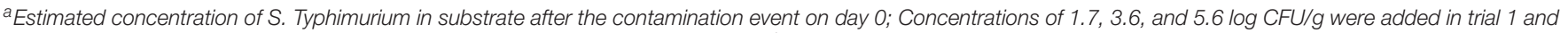

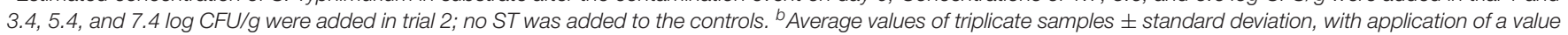

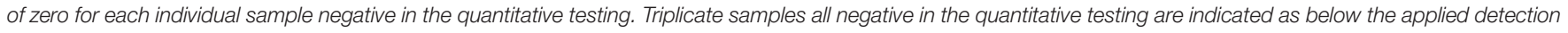

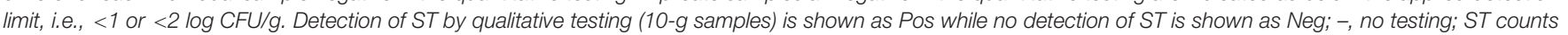

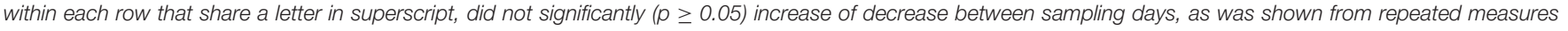
ANOVA.

"Qualitative Detection of S. Typhimurium." The quantitative detection of the ST contamination strain, showed that the detection level was close or similar to the level of cells added $(p=0.45)$, see Table 2 .

The added cell levels of 1.01 and $1.21 \log$ CFU/g in trials 1 and 2, respectively, are close to the theoretical detection level of $1 \log \mathrm{CFU} / \mathrm{g}$ when $1 \mathrm{~mL}$ of the $10^{-1}$ dilution is plated, which may explain the negative test-result obtained for $1.01 \mathrm{log}$ $\mathrm{CFU} / \mathrm{g}$ in trial 1.

For the qualitative detection method, $S$. Typhimurium was detected in 1 out of 2 larvae samples at a contamination level of $0.6 \mathrm{CFU}$ per $10 \mathrm{~g}$ sample, while both sample replicates were positive when the contamination level was 10 -fold higher, i.e., $6 \mathrm{CFU}$ or higher.

\section{Experimental Exposure of Mealworms to Salmonella-Contaminated Substrate}

Mealworms were at start of each of the two experimental periods exposed to substrate contaminated with $S$. Typhimurium in concentrations ranging from 1.7 to $7.4 \log \mathrm{CFU} / \mathrm{g}$. The quantitative level of ST found in larvae and substrate during the 14-day study period is shown as an average of triplicate samples ( $1 \mathrm{~g}$ each) in Table 3 . In case individual samples tested negative, i.e., below the detection limit of 1 or $2 \log \mathrm{CFU} / \mathrm{g}$, a value of zero was applied for calculation of the average.

The level of ST found in larvae was below the quantitative detection level in all three samples (in Table 3 shown as $<1$ or $<2 \log$ CFU/g) already within 1 day in larvae exposed to contamination levels of 1.7, 3.4, and 3.6 log CFU/g opposed to contamination levels of 5.4, 5.6, and 7.4 log CFU/g, respectively. The maximum level of ST detected in individual 1-g larvae samples (i.e., 8 larvae) was $5.8 \log \mathrm{CFU} / \mathrm{g}$ detected on day 0 . Beyond day 7, only larvae exposed to the highest contamination level, i.e., $7.4 \log \mathrm{CFU} / \mathrm{g}$ were $>1.0 \log \mathrm{CFU} / \mathrm{g}$, but often with variation of the ST level in individual larvae samples with findings of, e.g., <2.0, 2.4, and $4.4 \log \mathrm{CFU} / \mathrm{g}$ on day 9. At end of the experiment on day 14, the ST level in these larvae was $1.9 \pm 0.3 \log \mathrm{CFU} / \mathrm{g}$.

When testing of 1-g larvae samples (non-disinfected) from each experimental tray reached the detection limit, 10-g samples 
were tested qualitatively for the presence of ST in the succeeding samplings. This showed the presence of ST in non-disinfected larvae at all contamination levels until the end of the experiment on day 14 as indicated by Pos for positive in Table 3 (i.e., at least $1 \mathrm{CFU} / \mathrm{g}$ ) except for the lowest contamination level of $1.7 \mathrm{log}$ $\mathrm{CFU} / \mathrm{g}$. Here, the ST level was $<1.0 \log \mathrm{CFU} / \mathrm{g}$ on day 1 and no ST was found in three succeeding samplings on days 2,5 , and 7.

In the negative control tray where no ST was added, weekly testing of larvae and substrate showed absence of ST, i.e., no indication of cross-contamination between the experimental trays.

In this experiment as well as under natural rearing conditions, mealworms inhabits their substrate and therefore surface disinfection was applied in an attempt to disclose whether detected $S$. Typhimurium derived from ingested ST or merely from contamination on the surface of the larvae. The applied disinfection method with $70 \%$ ethanol and washing twice in MilliQ water had been tested prior to this study (see section "Surface Disinfection of Larvae"), where visual inspection of the larvae embedded in the agar plates showed no growth of ST on 20 out of the 21 larvae disinfected in total. ST was found on a single larva but with markedly lower growth of ST than on control larvae rinsed with water only, overall indicating efficacy of the applied disinfection method.

For surface disinfected larvae, ST was on day 0 detected in 15 out of 18 larvae samples and not in levels markedly different from non-disinfected larvae. However, at later samplings, the disinfected larvae generally rendered test-negative earlier than the untreated larvae, and only at the highest contamination level of $7.4 \mathrm{log} \mathrm{CFU} / \mathrm{g}$ was ST found present in disinfected larvae at the end of experiment on day 14 (see Table 3). The qualitative testing of disinfected larvae was performed less frequently not to skew the 1:1 ratio between substrate and larvae too much when removing samples of $10 \mathrm{~g}$, and further, it was considered superfluous if the non-disinfected counterpart tested negative already.

In the substrate, the level of $S$ Typhimurium on day 0 shortly after contamination was up to 1.4 log lower than the expected level of ST added (see Table 3). At the three lowest contamination levels (1.7-3.6 log CFU/g), the ST level in the substrate was below the detection limit on day 2 and onwards. Still, ST was found until the end of experiment by qualitative testing except for the low contamination level, i.e., $1.7 \mathrm{log} \mathrm{CFU} / \mathrm{g}$. At the contamination levels of 5.4, 5.6, and 7.4 log CFU/g, the level of ST detected in the substrate on day 14 was $2.3 \pm 0.2,2.0 \pm 2.0$, and $3.7 \pm 0.1 \log$ $\mathrm{CFU} / \mathrm{g}$, respectively.

The level of $S$. Typhimurium in substrate from trays without larvae (control) was compared with ST levels in the trays containing larvae at contamination levels of 3.6 and $5.4 \mathrm{log}$ CFU/g in trials 1 and 2, respectively (see Table 3 ). In the first trial, ST was generally below the quantitative detection level within the first days both in trays with and without larvae present although ST was detected until the end of the experiment. At the higher contamination level in the second trial, the ST level in the substrate remained quantifiable in trays with larvae, while the substrate without larvae after day 7 was ST-positive by preenrichment only.

\section{DISCUSSION}

In order to assess the level of Salmonella Typhimurium cells in mealworm (T. molitor) larvae after exposure to contaminated substrate, we made a preliminary test of the ability to recover $S$. Typhimurium from mealworm samples with the applied detection method. A standard approach for determination of bacterial cell numbers in a sample is spreading of a sample dilution series on agar plates. This approach though, has a theoretically limit of detection depending on the portion of sample plated. Further, the high bacterial load generally present in mealworms may challenge the specific detection of the $S$. Typhimurium target strain if outcompeted by the inherent bacteria (Vandeweyer et al., 2017; Wynants et al., 2018). Therefore, a S. Typhimurium strain with an antibiotic resistance marker (rifampicin) previously proven adequate for detection in pig fecal samples (Jensen et al., 2006) was chosen for the study to facilitate detection. Other strains of S. Typhimurium or other serovars may not have elicited the exact same outcome, however, $S$. Typhimurium is one of the most important serovars in human cases of salmonellosis and was considered a good candidate for the exposure study as a start.

Proper retrieval of bacterial cells from a sample is also important to obtain a good recovery of the bacteria of interest (Rohde et al., 2015). In this study, in-tube homogenization of mealworm samples by grinding with a sterile pestle tissue grinder was applied, as a small preliminary study indicated a good recovery of cells by this method as compared to stomaching and crushing by hands. This preliminary evaluation was based on total aerobic count of the inherent bacterial population (data not shown), as artificial spiking with the bacteria of interest is unlikely to reflect the natural binding or embedment of cells within a sample matrix. The in-tube homogenization approach appears convenient opposed to, e.g., blending or use of a mortar as there is no need for transferring the sample material after homogenization. Moreover, such transfer may lead to loss of material if the sample is weighed beforehand or failure in achieving a fully representative sample if weighing is performed on a sample not completely homogenous, which is likely for mealworms and other insects due to their exoskeleton parts.

The preliminary assessment of the detection limit for $S$. Typhimurium in mealworm samples indicated a good recovery of the cells added both for the quantitative estimation (Table 2) and the qualitative detection method applied in this study. The level of ST added in trial 1 was estimated to $1.01 \mathrm{log} \mathrm{CFU} / \mathrm{g}$, i.e., very close to the theoretical detection limit of $1 \mathrm{log} \mathrm{CFU} / \mathrm{g}$ when $1 \mathrm{~mL}$ of the $1: 9\left(10^{-1}\right)$ dilution is plated. So the lack of detection at this level seemed to be within the expected precision of the plate spreading method rather than suppression of ST growth, generally implying a low risk of incorrect conclusions due to false-negative test results.

In the experimental exposure study, the lowest contamination level of $1.7 \log \mathrm{CFU} / \mathrm{g}$ was the only one not resulting in ST positive mealworms at the end of the experiment, i.e., on day 14 for the non-disinfected larvae. In a study by 
Wynants et al. (2019), a Salmonella contamination level of $2 \log \mathrm{CFU} / \mathrm{g}$ also resulted in test-negative larvae on day 7 where the experiment ended. At a contamination level of $4 \log \mathrm{CFU} / \mathrm{g}$, those authors found 4 of 6 larvae replicates Salmonella-negative (qualitative testing only) on day 7 in one experiment, while Salmonella was $<1.0 \mathrm{log} \mathrm{CFU} / \mathrm{g}$ in another experiment. Similarly, in the current study, contamination levels slightly lower at 3.4 and $3.6 \mathrm{log} \mathrm{CFU} / \mathrm{g}$ resulted in Salmonella $<1.0 \log \mathrm{CFU} / \mathrm{g}$ and $<2.0 \mathrm{log} \mathrm{CFU} / \mathrm{g}$ on day 1 . A detection limit of $2 \mathrm{log} \mathrm{CFU} / \mathrm{g}$ was applied for samples expected to reach this level, but the obtained ST counts proved to be lower in some cases, and here a detection limit of 1 $\log \mathrm{CFU} / \mathrm{g}$ would have been more informative. Nevertheless, the non-disinfected larvae remained ST positive throughout the experimental period of 14 days. Even though this may imply a Salmonella presence as low as 1 CFU per gram, the ID50 of 7 CFU for causing infection emphasizes the significance of these findings (Teunis et al., 2010). It also supports the recommendations concerning heat treatment or other processing to ensure the food safety of mealworms, although no specific food safety criteria [Regulation (EC) No 2073/2005] for edible insects have been established (yet).

The surface disinfected larvae generally turned ST-negative somewhat earlier than the corresponding non-disinfected larvae, indicating that some ST resided on the surface only. Surprisingly though on day 0 , the ST levels were similar in the disinfected and non-disinfected larvae. Given efficacy of the disinfection method, this implies a rapid ingestion of substrate (Salmonella), as sample collection were started approximately $10 \mathrm{~min}$ after addition of the contaminated spent grain. To our knowledge, no other studies have made a parallel testing of disinfected and non-disinfected larvae in the same experiment to shed light on this. Inefficient disinfection could also explain the high ST counts on day 0 , and despite our promising pre-evaluation of the method based on $70 \%$ ethanol, results by Crippen and Sheffield (2006) indicated that $70 \%$ ethanol alone was inefficient for surface disinfection of beetles of the lesser mealworm (Alphitobius diaperinus). However, for their assessment, disinfected beetles were submerged completely into the growth medium, and although shortly, excretion of internal bacteria into the medium during the submersion cannot be excluded. Regardless, any detection of Salmonella either internal or external is of concern from a food safety perspective, as the whole larvae will be processed.

At a contamination level of 7 log CFU/g, Wynants et al. (2019) observed no decrease in the Salmonella level in larvae on day $7(4.1 \pm 1.1 \log$ CFU/g) and it was discussed whether it was simply a matter of longer time needed to exert a reduction or if numbers were too abundant for a reduction to happen. The only other contamination level tested in that experiment was 4 $\log \mathrm{CFU} / \mathrm{g}$, which resulted in $<1 \log \mathrm{CFU} / \mathrm{g}$ in larvae at day 7 , i.e., the fate of Salmonella at contaminations between 4 and 7 $\log \mathrm{CFU} / \mathrm{g}$ is uncertain. In our study, the ST counts for $7.4 \mathrm{log}$ $\mathrm{CFU} / \mathrm{g}$ were $5.2 \pm 0.5,3.1 \pm 1.2$, and $1.9 \pm 0.3 \mathrm{log} \mathrm{CFU} / \mathrm{g}$ on days 0,7 , and 14, respectively and at $5.4 \log \mathrm{CFU} / \mathrm{g}$, the ST counts were $<1.0 \log \mathrm{CFU} / \mathrm{g}$ on day 9 but ST positive until day 14 .
This indicates a decrease over time but also that the additional 7 days in the current study were insufficient for clearing the contamination in the larvae. Also Crippen et al. (2012) found that the lesser mealworm excreted Salmonella into their feces (frass) for 6-12 days after a $2 \mathrm{~h}$ exposure to $8 \log \mathrm{CFU} / \mathrm{mL}$, and where 33 and $<10 \%$ of the larvae shed Salmonella on day 9 and day 12, respectively. In that study, however, the larvae were isolated from the source of contamination after $2 \mathrm{~h}$, i.e., not reflecting real life rearing conditions where larvae inhabit their substrate, where contaminations may persist and constitute a continuous exposure. Although the ST counts found in our larvae depended on the initial contamination level, absence of ST within the 14-day course was not observed in non-disinfected larvae for contaminations levels $\geq 3.4 \mathrm{log} \mathrm{CFU} / \mathrm{g}$. So one can still pose the questions whether longer time ( $>14$ days) was needed to clear the contamination in the larvae or if there is a certain threshold ( $>2 \log \mathrm{CFU} / \mathrm{g}$ based on our study and Wynants et al., 2019) above which Salmonella will persist until harvest prior to pupation. Further, the answers may depend on several other factors like type of substrate, larval density or the larval stage at which the contamination event occurs, as younger larvae may elicit less colonization resistance to foreign microorganisms (Wynants et al., 2019). Moreover, it is uncertain how the design of the experimental set-up affects the results. For example, the volume of Salmonella suspension chosen for contaminating the substrate will affect the moisture content and hence growth potential of bacteria, while the method of distribution may influence the actual level of contamination experienced by individual larvae.

Concerning the current experimental design, the ST was added via the wet substrate (spent grain) to avoid clumping of the dry flour-based substrate. The spent grain constituted $12 \%$ of the substrate in total, and despite mixing efforts it may have caused an uneven distribution of ST and partly explain the discrepancy (up to $1.4 \mathrm{log}$ ) between the ST counts obtained on day 0 and the ST numbers added (Table 3). However, Salmonella contaminations occurring under natural rearing conditions will most likely result in a heterogeneous distribution of cells as well, and although larvae activity probably aid a more homogeneous distribution of contaminants over time, the triplicate substrate and larvae samples had variable ST counts as evident from the standard deviations of the averages. This possible variation between samples will also need consideration when sampling for monitoring purposes.

In substrate contaminated at levels $\leq 3.6 \mathrm{log} \mathrm{CFU} / \mathrm{g}, \mathrm{ST}$ counts were soon below the detection limit, although only STnegative on day 14 for the lowest contamination level (1.7 $\log$ CFU/g) as the case for the larvae. At the 5.4 and $5.6 \log$ CFU/g contamination levels, the ST counts in substrate were reduced to $2.3 \pm 0.2$ and $2.0 \pm 2.0$ on day 14 , respectively, but counts were not significantly different from the initial ST level counted on day 0 (Table 3 ). Noticeable, although the ST contamination level found in substrate on day 14 in these trays was close to the initial contamination level of $1.7 \mathrm{log}$ $\mathrm{CFU} / \mathrm{g}$ where larvae samples rendered ST-negative within the first days, the larvae in these trays were still ST-positive on day 14. Similarly, the initial $7.4 \mathrm{log} \mathrm{CFU} / \mathrm{g}$ contamination level in 
substrate decreased significantly to $3.7 \pm 0.1 \log$ CFU/g on day 14 , but this level was sufficient for ST counts reaching $1.9 \pm 0.3 \log$ $\mathrm{CFU} / \mathrm{g}$ in the larvae opposed to trays with initial contamination levels of 3.4 and $3.6 \mathrm{log} \mathrm{CFU} / \mathrm{g}$. All together indicating the importance of the initial contamination level in the substrate for the resulting ST counts in the larvae. Still, it is uncertain for how long Salmonella will remain present at contamination levels $>2 \log \mathrm{CFU} / \mathrm{g}$ as only contamination levels $\leq 2 \log \mathrm{CFU} / \mathrm{g}$ resulted in absence of ST at the end of experiments. Further, this study was based on a single contamination event while it is uncertain if a repetitious introduction of Salmonella might support longer persistence of Salmonella in the larvae even at low contaminations levels.

Wynants et al. (2019) found that Salmonella survived well in the wheat bran without larvae during the experimental period of 7 days. Further, the substrate without larvae had higher Salmonella counts or more Salmonella-positive replicates than substrate with larvae, which indicated that the larvae supported reduction of Salmonella. Contrary, our results for the contamination level of $5.4 \mathrm{log} \mathrm{CFU} / \mathrm{g}$ indicated that ST counts in substrate were higher when larvae were present. Irrespective, at least no proliferation of Salmonella was observed in neither substrate nor larvae in both studies. For substrate, which is mainly dry, this may be explained by a required water activity $a_{\mathrm{w}}$ level of minimum 0.93 to facilitate growth of Salmonella, while Salmonella easily survive under dry conditions (Podolak et al., 2010). However, the humid rearing conditions for mealworms (often $50-70 \% \mathrm{RH}$ ) and addition of wet substrate as water source can be suspected to create 'wet spots' facilitating growth. No $a_{\mathrm{w}}$ measurements of the contaminated substrate were conducted in this study, but previous in-house measurements had indicated an $a_{\mathrm{w}}$ of $0.861 \mathrm{~h}$ after addition of spent grain in rearing boxes, i.e., well below the growth supportive level. So it is uncertain if the higher ST counts observed in substrate with larvae in our study were partly due to the continuous addition of spent grain increasing the moisture content, opposed to the single addition to trays without larvae to prevent molding. Further, as the gastrointestinal tract of the larvae provides humid conditions, it can be speculated if the passage of Salmonella through the larval gut is actually conducive for their survival after excretion considering the higher level of Salmonella found in substrate with larvae. The $\mathrm{pH}$ is 5.6 in the anterior and middle midgut of T. molitor larvae and 7.9 in the posterior midgut, i.e., there is no significant gastric acid barrier in the larvae acting on Salmonella that tolerate $\mathrm{pH}$ down to 4 (Moreira et al., 2017). This is in contrast to $\mathrm{pH}$ values as low as 2.0 and 3.1 in the middle midgut of larvae of black soldier flies (Hermetica illucens) and house flies (Musca domestica), respectively, assumingly reducing the change of Salmonella surviving passage through these fly larvae (Terra and Regel, 1995; Bonelli et al., 2019). Still, in T. molitor as in other insects, are antimicrobial peptides (AMP) reported to act in the defense against bacterial infections (Wu et al., 2018; Jo et al., 2019; Keshavarz et al., 2020). Nevertheless, Crippen et al. (2012) found that larvae of lesser mealworm excreted live Salmonella into their frass.

\section{CONCLUSION}

Considering the long-time efforts made for optimizing and standardizing bacteriological culturing protocols for monitoring and safety control of other food and feed production chains, similar standardization of methods for monitoring of insect production or products would help to ensure validity and comparability of test results within this completely new area. Not least if specific hygiene process criteria or food safety criteria for edible insects are to be established in future.

This study indicated the importance of avoiding introduction of Salmonella into the mealworm production site, e.g., via contaminated substrate in order to avoid Salmonella-positive larvae as they remained positive for at least 14 days when the initial contamination levels were $\geq 3.4 \log \mathrm{CFU} / \mathrm{g}$. However, this study was based on a single contamination event and the impact of repetitious introduction of Salmonella should be assessed to neglect contaminations $<2 \log \mathrm{CFU} / \mathrm{g}$. The initial contamination level affected the resulting Salmonella count in both larvae and substrate, and although the Salmonella level generally decreased over time and no proliferation of Salmonella was observed, proper treatment before consumption would be needed to ensure the food safety of larvae.

\section{DATA AVAILABILITY STATEMENT}

The raw data supporting the conclusions of this article will be made available by the authors, without undue reservation, to any qualified researcher.

\section{AUTHOR CONTRIBUTIONS}

$\mathrm{AJ}$ and $\mathrm{SH}$ contributed design and conductance of study and data analysis. AJ wrote the first draft of the manuscript and performed statistical analysis. DB and AJ contributed conception and management of the project. All authors contributed to discussion of results, manuscript revision, and read and approved the submitted version.

\section{FUNDING}

This work was conducted as part of the inVALUABLE (Insect Value Chain in a Circular Bioeconomy) project funded by Innovation Fund Denmark under Grand Solutions No. 615000011B.

\section{ACKNOWLEDGMENTS}

Thanks to Adival A/S for providing the substrate for the mealworms and DTU Brewery for the spent grain and Emmalie Thøtt Strand for technical assistance in the lab. 


\section{REFERENCES}

Blazar, J. M., Lienau, E. K., and Allard, M. W. (2011). Insects as vectors of foodborne pathogenic bacteria. Terr. Arthropod Rev. 4, 5-16. doi: 10.1163/ 187498311 X543989

Bonelli, M., Bruno, D., Caccia, S., Sgambetterra, G., Cappellozza, S., Jucker, C., et al. (2019). Structural and functional characterization of Hermetia illucens larval midgut. Front. Physiol. 10:204. doi: 10.3389/fphys.2019.00204

Cappelli, A., Cini, E., Lorini, C., Oliva, N., and Bonaccorsi, G. (2020). Insects as food: a review on risks assessments of Tenebrionidae and Gryllidae in relation to a first machines and plants development. Food Control 108:106877. doi: 10.1016/j.foodcont.2019.106877

Crippen, T. L., and Sheffield, C. (2006). External surface disinfection of the lesser mealworm (Coleoptera: Tenebrionidae). J. Med. Entomol. 43, 916-923.

Crippen, T. L., Sheffield, C. L., Beier, R. C., and Nisbet, D. J. (2018). The horizontal transfer of Salmonella between the lesser mealworm (Alphitobius diaperinus) and poultry manure. Zoonoses Public Health 65, e23-e33. doi: 10.1111/zph. 12404

Crippen, T. L., Zheng, L., Sheffield, C. L., Tomberlin, J. K., Beier, R. C., and $\mathrm{Yu}, \mathrm{Z}$. (2012). Transient gut retention and persistence of Salmonella through metamorphosis in the lesser mealworm, Alphitobius diaperinus (Coleoptera: Tenebrionidae). J. Appl. Microbiol. 112, 920-926. doi: 10.1111/j.1365-2672.2012. 05265.x

EFSA Scientific Committee (2015). Risk profile related to production and consumption of insects as food and feed. EFSA J. 13:4257. doi: 10.2903/j.efsa. 2015.4257

European Food Safety Authority [EFSA], and European Centre for Disease Prevention, and Control [ECDC] (2019). The European Union One Health 2018 Zoonoses Report. EFSA J. 17:5926. doi: 10.2903/j.efsa.2019.5926

Garofalo, C., Milanoviæ, V., Cardinali, F., Aquilanti, L., Clementi, F., and Osimani, A. (2019). Current knowledge on the microbiota of edible insects intended for human consumption: a state-of-the-art review. Food Res. Int. 125:108527. doi: $10.1016 /$ j.foodres.2019.108527

Grabowski, N. T., and Klein, G. (2017). Microbiological analysis of raw edible insects. J. Insects as Food Feed 3, 7-14. doi: 10.3920/JIFF2016.0004

Grimont, P. A., and Weill, F.-X. (2007). Antigenic Formulae of the Salmonella serovars, 9th Edn. Paris: WHO Collaborating Centre for Reference and Research on Salmonella.

Imathiu, S. (2020). Benefits and food safety concerns associated with consumption of edible insects. NFS J. 18, 1-11. doi: 10.1016/j.nfs.2019.11.002

ISO (2017). Microbiology of the Food Chain - Horizontal Method for the Detection, Enumeration and Serotyping of Salmonella - Part 1: Detection of Salmonella spp. (ISO 6579-1, 2017). Geneva: ISO.

Jensen, A. N., Dalsgaard, A., Stockmarr, A., Nielsen, E. M., and Baggesen, D. L. (2006). Survival and transmission of Salmonella enterica serovar typhimurium in an outdoor organic pig farming environment. Appl. Environ. Microbiol. 72, 1833-1842. doi: 10.1128/AEM.72.3.1833-1842.2006

Jo, Y. H., Patnaik, B. B., Hwang, J., Park, K. B., Ko, H. J., Kim, C. E., et al. (2019). Regulation of the expression of nine antimicrobial peptide genes by TmIMD confers resistance against Gram-negative bacteria. Sci. Rep. 9:10138 doi: 10.1038/s41598-019-46222-8

Keshavarz, M., Jo, Y. H., Patnaik, B. B., Park, K. B., Ko, H. J., Kim, C. E., et al. (2020). TmRelish is required for regulating the antimicrobial responses to Escherichia coli and Staphylococcus aureus in Tenebrio molitor. Sci. Rep 10:4258. doi: 10.1038/s41598-020-61157-1

Moreira, N. R., Cardoso, C., Dias, R. O., Ferreira, C., and Terra, W. R. (2017). A physiologically-oriented transcriptomic analysis of the midgut of Tenebrio molitor. J. Insect Physiol. 99, 58-66. doi: 10.1016/j.jinsphys.2017.03.009

Nordentoft, S., Fischer, C., Bjerrum, L., Heckmann, L. H., and Hald, B. (2017). Reduction of Escherichia coli, Salmonella Enteritidis and Campylobacter jejuni in poultry manure by rearing of Musca domestica fly larvae. I. Insects Food Feed 3, 145-153. doi: 10.3920/JIFF2016.0058

Osimani, A., Milanoviæ, V., Cardinali, F., Garofalo, C., Clementi, F., Pasquini, M., et al. (2018). The bacterial biota of laboratory-reared edible mealworms (Tenebrio molitor L.): from feed to frass. Int. J. Food Microbiol. 272, 49-60. doi: 10.1016/j.ijfoodmicro.2018.03.001

Podolak, R., Enache, E., Stone, W., Black, D. G., and Elliott, P. H. (2010). Sources and risk factors for contamination, survival, persistence, and heat resistance of
Salmonella in low-moisture foods. J. Food Prot. 73, 1919-1936. doi: 10.4315/ 0362-028X-73.10.1919

Raheem, D., Raposo, A., Oluwole, O. B., Nieuwland, M., Saraiva, A., and Carrascosa, C. (2019). Entomophagy: nutritional, ecological, safety and legislation aspects. Food Res. Int. 126:108672. doi: 10.1016/j.foodres.2019. 108672

Roche, A. J., Cox, N. A., Richardson, L. J., Buhr, R. J., Cason, J. A., Fairchild, B. D., et al. (2009). Transmission of Salmonella to broilers by contaminated larval and adult lesser mealworms, Alphitobius diaperinus (Coleoptera: Tenebrionidae). Poult. Sci. 88, 44-48. doi: 10.3382/ps.2008-00235

Rohde, A., Hammer, J. A., Appel, B., Dieckmann, R., and Dahouk, A. L. (2015). Sampling and homogenization strategies significantly influence the detection of foodborne pathogens in meat. J. Biomed. Biotech. 2015, 1-8. doi: 10.1155/ 2015/145437

Schlüter, O., Rumpold, B., Holzhauser, T., Roth, A., Vogel, R. F., Quasigroch, W., et al. (2016). Safety aspects of the production of foods and food ingredients from insects. Mol. Nutr. Food Res. 61:1600520. doi: 10.1002/mnfr.201600520

Skov, M. N., Spencer, A. G., Hald, B., Petersen, L., Nauerby, B., Carstensen, B., et al. (2004). The role of litter beetles as potential reservoir for Salmonella enterica and thermophilic Campylobacter spp. between broiler flocks. Avian Dis. 48, 9-18. doi: 10.1637/5698

Stoops, J., Crauwels, S., Waud, M., Claes, J., Lievens, B., and Van Campenhout, L. (2016). Microbial community assessment of mealworm larvae (Tenebrio molitor) and grasshoppers (Locusta migratoria migratorioides) sold for human consumption. Food Microbiol. 53, 122-127. doi: 10.1016/j.fm.2015. 09.010

Terra, W. R., and Regel, R. (1995). pH buffering in Musca domestica midguts. Comp. Biochem. Physiol. Part A: Physiol. 112, 559-564. doi: 10.1016/03009629(95)02028-4

Teunis, P. F. M., Kasuga, F., Fazil, A., Ogden, I. D., Rotariu, O., and Strachan, N. J. C. (2010). Dose-response modeling of Salmonella using outbreak data. Int. J. Food Microbiol. 144, 243-249. doi: 10.1016/j.ijfoodmicro.2010 .09 .026

van der Fels-Klerx, H. J., Camenzuli, L., Belluco, S., Meijer, N., and Ricci, A. (2018). Food Safety Issues Related to Uses of Insects for Feeds and Foods. Comp. Rev. Food Sci. Food Saf. 17, 1172-1183. doi: 10.1111/1541-4337.12385

van Huis, A., Van Itterbeeck, J., Klunder, H., Mertens, E., Halloran, A., Muir, G., et al. (2013). Edible Insects - Future Prospects for Food and feed Security. FAO Forestry Paper 171. Rome: Food and Agriculture Organization of the United Nations.

Vandeweyer, D., Crauwels, S., Lievens, B., and Van Campenhout, L. (2017). Microbial counts of mealworm larvae (Tenebrio molitor) and crickets (Acheta domesticus and Gryllodes sigillatus) from different rearing companies and different production batches. Int. J. Food Microbiol. 242, 13-18. doi: 10.1016/ j.ijfoodmicro.2016.11.007

Wu, Q., Patoèka, J., and Kuèa, K. (2018). Insect antimicrobial peptides, a mini review. Toxins 10:461. doi: 10.3390/toxins10110461

Wynants, E., Crauwels, S., Verreth, C., Gianotten, N., Lievens, B., Claes, J., et al. (2018). Microbial dynamics during production of lesser mealworms (Alphitobius diaperinus) for human consumption at industrial scale. Food Microbiol. 70, 181-191. doi: 10.1016/j.fm.2017.09.012

Wynants, E., Frooninckx, L., Van Miert, S., Geeraerd, A., Claes, J., and Van Campenhout, L. (2019). Risks related to the presence of Salmonella sp. during rearing of mealworms (Tenebrio molitor) for food or feed: survival in the substrate and transmission to the larvae. Food Control 100, 227-234. doi: 10. 1016/j.foodcont.2019.01.026

Conflict of Interest: The authors declare that the research was conducted in the absence of any commercial or financial relationships that could be construed as a potential conflict of interest.

Copyright (c) 2020 Jensen, Hansen and Baggesen. This is an open-access article distributed under the terms of the Creative Commons Attribution License (CC BY). The use, distribution or reproduction in other forums is permitted, provided the original author(s) and the copyright owner(s) are credited and that the original publication in this journal is cited, in accordance with accepted academic practice. No use, distribution or reproduction is permitted which does not comply with these terms. 